\title{
HAMBRE, MORTALIDAD Y CRECIMIENTO DEMOGRAFICO EN LAS POBLACIONES DE LA EUROPA PREINDUSTRIAL *
}

\author{
VICENTE PEREZ MOREDA \\ Universidad Complutense
}

«¿Se morian realmente de hambre los campesinos?» Esta es la pregunta que se hacía Peter Laslett al frente de uno de los capítulos de su clásica obra El mundo que bemos perdido. La respuesta contundente era no, al menos de forma regular o en proporciones significativas, y al menos en el caso inglés'. Por lo que ya se sabe de otras zonas del continente europeo, la respuesta debería ser similar, con matizaciones también muy parecidas. El hambre pura, como factor estrictamente aislado de mortalidad, no deja de tener un carácter marginal en el conjunto de posibles causas de la elevada mortalidad del pasado. Frente a posiciones clásicas como la de P. Goubert, quien en 1952 afirmaba que las epidemias «puras», sin relación con una carestía de los cereales, no parecían haber ejercido una influencia determinante sobre la población ${ }^{2}$, los historiadores han ido destacando posteriormente la responsabilidad del factor epidémico, independiente de la coyuntura económica, en muchas de las crisis demográficas del pasado ${ }^{3}$. El mismo Goubert hace tiempo que matizó ya sus antiguas opiniones al respecto ${ }^{4}$, mientras que otros especialistas, como J. Dupâquier, se sitúan radicalmente en el extremo opuesto, al afirmar que «el elemento constitutivo de toda crisis es la epidemia, sin la cual la carestía apenas podría mostrar sus efectos sobre la mortalidad ${ }^{5}$.

\footnotetext{
* Algunas de las reflexiones que siguen fueron expuestas en el seminario que tuvo lugar en la Fundación Ortega y Gasset (Madrid), en mayo de 1987, dedicado al examen de la última obra de Massimo Livi Bacci, y al que se refiere el autor en el Prefacio de la edición española de su libro (p. 11).

' En la última versión, ampliamente revisada, de esta obra, el capítulo 6, que lleva ese título, tiene mucho que ver con los temas que discute Livi Bacci en el libro que es objeto de atención en estas páginas. Véase Peter Laslett (1983), pp. 122-152.

${ }^{2}$ Pierre Goubert (1952), pp. 453-468.

${ }^{3}$ Véanse, por ejemplo, Andrew B. Appleby (1973), pp. 403-432, y (1977), pp. 508-512; Jean Noël Biraben (1975-76); John D. Post (1976), pp. 14-37; y Sölvi Sogner (1979).

4 En unas «reflexiones posteriores», firmadas en $1974 \mathrm{e}$ incluidas en la reedición del artículo anterior, reconocía que «trabajos posteriores han demostrado claramente que el elemento epidémico contaba mucho más de lo que yo creía entonces», P. Goubert (1976), pp. 158-159.
}

s Jacques Dupâquier (1979), p. 18. 
A pesar de ello, subsiste aún la arraigada opinión de que el hambre, o la desnutrición crónica, constituían uno de los principales agentes responsables de las altas tasas de mortalidad, aunque sólo fuera por la frecuencia y la intensidad de las crisis demográficas que generaban o contribuían a agudizar. Por lo tanto, el factor alimentario, a través de la mortalidad, sigue siendo utilizado muy a menudo como el principal elemento explicativo de los grandes ciclos demográficos de la historia preindustrial europea.

El libro de Livi Bacci Ensayo sobre la bistoria demográfica europea, que en una edición bastante deplorable apareció traducido al castellano hace unos meses ${ }^{6}$, intenta combatir la persistencia de ese mito del hambre o la desnutrición como claves explicativas de la mortalidad y el crecimiento demográfico en la Europa preindustrial. En palabras del mismo autor, «este ensayo trata el tema de las interdependencias de alimentación, supervivencia y población, y la hipótesis alimentaria se somete a verificación, buscando pruebas e indicios en los nutridos campos de la biología y de la epidemiología, al igual que en la historia económica y en la social» (p. 9). Livi Bacci encuentra muchas más razones para refutar esa hipótesis que para confirmarla.

Hay tanta insistencia en muchos de los argumentos que se exponen, y tanta acumulación de pruebas en contra de la «hipótesis alimentaria», que una impresión rápida conduciría a pensar que lo que se pretende es negar cualquier vínculo entre el hambre $\multimap$ los niveles de nutrición en general- y la mortalidad y el crecimiento demográfico. Sin embargo, los juicios del autor y las conclusiones de la obra están mucho más matizados y, en lo sustancial, no se desvían de la opinión generalizada hoy día entre la comunidad científica, algunos de cuyos ejemplos he citado anteriormente. En cualquier caso, el mayor interés y la gran novedad del último libro de Livi Bacci reside no sólo en su lucha demoledora contra el empleo abusivo y precipitado del factor "hambre» en muchas de las explicaciones del crecimiento demográfico, sino en su análisis ponderado y minucioso de uno de los integrantes del «síndrome de atraso", a saber, la desnutrición, cuyo poder explicativo, por sí mismo, tal vez no sea tan relevante como a veces se supone, al menos en la experiencia histórica sometida a examen. Aunque sólo fuera por ello, la obra merecería figurar como un hito en la literatura demográfica de nuestro tiempo, sobre todo si tenemos en cuenta que el tipo de análisis que el autor diseña y la concepción interdisciplinaria que incorpora van a permitir un enfoque mucho más riguroso de los problemas que plantea, incluso aunque algunos puntos con-

- Con el subtítulo, más revelador de su contenido, de Población y alimentación en Europa. A decir verdad, el título original de la edición italiana (Popolazione e alimentazione tra antagonismo e adattamento) tampoco ayudaba mucho al editor de la versión española. 
cretos de la argumentación, o algunas conclusiones parciales, sean objeto de crítica u origen de alguna polémica.

En el primer capítulo, de carácter introductorio, se ofrece un panorama del crecimiento demográfico a largo plazo, junto a una discusión de las diversas interpretaciones que se han dado del mismo, momento que el autor aprovecha para explicar las relaciones entre recursos alimentarios y población dentro de su personal lectura del modelo malthusiano. Entre los factores constrictivos que a través de la mortalidad han condicionado dicho crecimiento, la atención se dirige a las variaciones en el régimen alimentario, del que se señalan tres «revoluciones» -en el Neolítico, la Baja Edad Media y el siglo Xviı - ninguna de las cuales —salvo quizá la última de ellas, como ahora se verá- fue acompañada de paralelos avances en los índices generales de supervivencia de la población.

$\mathrm{El}$ autor inicia aquí un ataque sistemático a quienes sostienen la relevancia de los niveles de nutrición en el ciclo demográfico, tesis defendida principalmente, y en lo que se refiere al ciclo demográfico moderno, que se inicia a mediados del siglo xviri, por Thomas McKeown ${ }^{7}$. Y es ahora cuando empiezan a surgir algunos puntos discutibles en el razonamiento de Livi Bacci. Es cierto que la población, en muchas zonas de Europa, creció en ese siglo sin conocer notables mejoras alimentarias (aunque deberíamos conocer mejor la relación entre los cambios agrarios y los niveles de nutrición reales, sin olvidar que hubo importantes procesos de roturación generalizados por casi todo el continente, una gran difusión de nuevas plantas como la patata e importantes cambios en la regulación del comercio exterior y en la distribución de granos, incluso allí donde no hubo rastro alguno de algo parecido a un «sistema Norfolk»). También podemos admitir que, en el caso inglés, fueron las variaciones en la nupcialidad y la natalidad, más que las de la mortalidad, las que mejor explican el crecimiento en ese período. Pero, aun así, Livi Bacci reco. noce que, al menos en el caso de los tres países europeos cuyo movimiento demográfico mejor conocemos - Gran Bretaña, Francia y Suecia-, «la mortalidad disminuye de manera sensible» en el siglo xviIr y a comienzos del siglo siguiente, «... y este hecho parece valorizar la tesis de McKeown». La relación causal entre los cambios en la nutrición y en la mortalidad no le parece clara, sin embargo, pues es posible que unas mayores disponibilidades alimentarias permitieran a la población «crecer pero no mejorar el nivel nutritivo». En muchos casos, como el irlandés, «el incremento demográfico llevaba al deterioro del nivel de nutrición» (pp. 39-40).

Lo que está claro, sin embargo, y es expresamente admitido en esos pá-

7 Thomas McKeown (1976). Una defensa más reciente de estas tesis, en Th. McKeown (1985). 
rrafos, es la existencia, en diversas zonas europeas al menos, de esa «tercera revolución alimentaria», a la que siguieron un "sensible» descenso de la mortalidad y un paralelo crecimiento demográfico. Esa es precisamente la tesis de McKeown, quien también se apoya, por lo demás, en la experiencia de los tres países citados. Otra cosa es el posible deterioro en la calidad de la alimentación con que cuenta una población en crecimiento y que genera una demanda cada vez mayor de subsistencias. Habría que ver si el crecimiento de la oferta, en términos cuantitativos, compensa o no ese posible deterioro en términos cualitativos: al fin y al cabo, la tragedia demográfica irlandesa no fue producto de la mediocre calidad de la dieta de su población (que, ciertamente, no era completa ni equilibrada), sino de un brusco descenso del volumen de la oferta. En todo caso, parece contradictorio referirse al período de finales del siglo xviri y comienzos del xIx admitiendo al mismo tiempo la existencia de una «revolución alimentaria» y de un posible «deterioro del nivel de nutrición».

Este capítulo de la obra concluye con una certera sentencia, que nos recuerda la dificultad del tema y nos pone en guardia frente a conclusiones supuestamente definitivas: «los mecanismos que unen demografía y alimentación son bastante ambiguos, y las simplificaciones no favorecen la claridad» (p. 40).

En el capítulo siguiente se establece, de forma precisa y documentada, un nivel mínimo de nutrición por debajo del cual es posible que empezaran a aumentar los riesgos de mortalidad. Dicho «umbral de peligro» se fija, para poblaciones históricas del período considerado, en una dieta diaria habitual de unas 2.000 calorías per capita $( \pm 10$ por 100$)$, que pueden ser, además, de origen muy diverso, pues hay que recordar que dietas básicamente vegetarianas, como la mediterránea, pueden satisfacer no sólo las exigencias energéticas, sino asegurar también las dosis requeridas de los principios nutritivos esenciales: una adecuada combinación de cereales, legumbres y verduras contiene, por ejemplo, las proteínas exigibles en una dieta ideal.

Se examinan después, en ese mismo capítulo, las complejas relaciones entre nutrición, infección y mortalidad, estudiadas en una amplísima literatura biomédica, de la que se da buena cuenta en esas páginas. El dato fundamental que subrayan la práctica totalidad de los especialistas es el de la relación reforzada o «sinérgica» entre la malnutrición y el agravamiento de muchos procesos de infección, relación que es recíproca y puede operar en ambas direcciones. El autor se acoge, en primer lugar, a las excepciones con que se enfrenta dicha asociación, algunas de las cuales son muy conocidas. Reproduce para ello un cuadro elaborado en la conferencia de Bellagio de 1982, que señala algunos de los principales procesos infecciosos cuya evolución puede verse muy influida por un estado deficitario de nutrición, frente a otros —en- 
tre los cuales cabe situar a cuadros patológicos como la viruela o la peste, causantes de una altísima mortalidad en el pasado- donde tal influencia es dudosa $o$ incluso mínima o inexistente ${ }^{8}$. Cabría plantear ciertas dudas res. pecto a la relación de algunas de estas enfermedades con los niveles de nutrición. Así, por ejemplo, no parece seguro que en el desárrollo clínico de la malaria tenga "poca o ninguna responsabilidad la insuficiencia alimentaria» (p. 62) ${ }^{9}$. La influencia de la malnutrición sobre el curso del tifus se valora como «incierta o variable», aludiéndose a experiencias históricas recientes (p. 63). Se podrían haber citado otros muchos testimonios históricos en sentido contrario, aunque es preciso admitir que una valoración rigurosa de los mismos - al igual que en el caso de la malaria - habría resultado muy difícil; de cualquier forma, el mismo Livi Bacci reconoce que «es plausible que el estado de subnutrición agrave el desarrollo del tifus» (p. 63). Tampoco está debidamente justificado que se califique de "contradictoria» la relación «entre incidencia de las enfermedades respiratorias y niveles de alimentación» (p. 64), sobre todo si pensamos en tipos concretos de infección, como la tuberculosis pulmonar, y si se tiene en cuenta que en el mismo cuadro que se incluye en el texto «la mayor parte de las infecciones respiratorias» figuran entre los procesos patológicos claramente influidos por los niveles de nutrición.

No está de más, en cualquier caso, recordar esas importantes excepciones a los vínculos entre malnutrición y desarrollo de los procesos de infección, para evitar interpretaciones simplistas de la mortalidad que frecuentemente se hacen con demasiada ligereza, y con el falso fundamento de una supuesta asociación automática y universal en aquel sentido. Sería conveniente, de todas formas, precisar lo más posible la relación entre los niveles de alimentación y el riesgo de muerte en los distintos procesos de infección, una vez que éstos han alcanzado una determinada prevalencia en la población. No es fácil distinguir la influencia de la desnutrición sobre el origen de la infección propiamente dicho de la que pueda existir sobre el agravamiento de la enfermedad ya contraída. Parece claro que el primer estadio del proceso de infección, el riesgo de contagio, es ajeno a los niveles de nutrición en muchos casos, pero

- Bellagio Conference (1982).

' No sólo los testimonios históricos aseguran que el mejor remedio contra la tercianas era «la quina y buen alimento" - véase esta cita y otras parecidas en Vicente Pérez Moreda (1980), p. 367-; también la experiencia contemporánea en la lucha por la erradicación del paludismo se ha mostrado a veces más eficaz con simples mejoras de la nutrición en las zonas afectadas que a través de medidas alternativas de saneamiento o terapia propiamente dicha -S. A. Meegama (1967)-. Por otra parte, estudios clásicos mostraron ya hace mucho tiempo que, si bien la infección palúdica es el origen de importantes trastornos nutritivos en los enfermos de menor edad, la persistencia de un estado de desnutrición agrava el curso de la enfermedad y dificulta su curación, ya que «en organismos debilitados y mal alimentados las recidivas se suceden con inusitada frecuencia», A. Barbosa y B. López Arjona (1935), pp. 59-65. 
no es seguro que sean tantas las enfermedades que no agravan su curso clínico en concurrencia con un estado de desnutrición, que puede ser provocada - o se ve incrementada - por la simple presencia de la infección.

En efecto, aun manteniéndose inalterables los niveles habituales de consumo, toda infección afecta negativamente al estado de nutrición del organismo. Y, por lo tanto, todo proceso de convalecencia requiere una mayor aportación proteínica y calórica para reponer las reservas consumidas en el curso de la infección ${ }^{10}$. Sin una mejora sustancial en la cantidad y la calidad de la dieta durante el período de convalecencia, es muy difícil salir de esa «espiral descendente» en el estado de salud " acción «sinérgica» entre infección y nutrición y que conducirá inevitablemente a aumentar la letalidad, el riesgo de mortalidad, de un determinado proceso infeccioso. Por todo ello, las relaciones posibles entre nutrición, infección y mortalidad podrían quedar mucho mejor definidas mostrando, por un lado, la vinculación entre desnutrición y morbilidad $\multimap$ incidencia- de una determinada patología y, por otro, entre desnutrición y letalidad de ese mismo proceso infeccioso. Pero la distinción entre los conceptos de morbilidad y letalidad no aparece en las páginas que ahora comentamos (a no ser, implícitamente, en la p. 61), y ello tal vez pudiera restar precisión a los argumentos que se exponen.

El capítulo siguiente examina el protagonismo de la carestía y el hambre en las crisis de mortalidad del pasado, con el objeto de someter a prueba la «hipótesis alimentaria». Algunos experimentos modernos y ciertos casos dramáticos también próximos a nosotros sirven para recordarnos los variados mecanismos de que dispone el organismo humano para adaptarse a una situación de estrés nutritivo. Huelgas de hambre mantenidas con absoluto rigor permitieron a sus protagonistas sobrevivir de dos a tres meses sin probar alimento alguno. Seis meses de semiayuno, con una reducción drástica de la dieta habitual, hacen aparecer, por otra parte, los síntomas del hambre, pero el organismo se ajusta a un nuevo equilibrio energético mediante la reducción del peso y de la actividad física, lo que supone un importante ahorro de consumo calórico. Situaciones bastante prolongadas de ayuno absoluto o de drástica reducción del consumo parece, pues, que pueden ser sobrellevadas «sin comprometer demasiado las posibilidades de supervivencia», y sin negativas consecuencias posteriores para el organismo (p. 69). Es útil recordar estos datos para comprender cómo la simple capacidad fisiológica de adaptación a una brusca reducción del consumo permitiría en muchas ocasiones «resistir un asedio, esperar la llegada de socorros y recoger una nueva cosecha» (p. 78).

"Ann G. Carmichael (1985), p. 51.
" Carl E. Taylor (1985), p. 289. 
No es menos cierto, sin embargo, que esos ejemplos, de duración relativamente prolongada, pero aislados en el contexto social de su época y únicos en la experiencia vital de sus protagonistas, tienen poco que ver - como parece admitirse en la p. 73 - con las experiencias históricas de escasez y semiayuno forzoso a las que se verían sometidos periódicamente nuestros antepasados, para algunos de los cuales debían de constituir más una situación habitual que un accidente ocasional. Como dice Livi Bacci algo más adelante, cuando empieza a analizar la mortalidad relacionada con las crisis de subsistencias en la Europa preindustrial, «la carestía es familiar en Europa, golpea repetidamente a cada generación en el curso de la vida, y de estas repetidas experiencias de estrés nutritivo de masa podemos esperar una información preciosa sobre las relaciones entre alimentación y mortalidad» (p. 79) ${ }^{12}$.

Ante la simultaneidad con que solían presentarse, en una amplia fase histórica bien documentada, las grandes hambrunas y muchas de las más importantes crisis de mortalidad, coincidentes también, en muchos casos, con la explosión de grandes epidemias, cabe preguntarse si epidemias y elevaciones catastróficas de la mortalidad estaban vinculadas de alguna forma con una coyuntura alimentaria desfavorable. Una «breve» pero detallada cronología de las grandes carestías que padeció la Europa preindustrial, y un examen de las vicisitudes de la mortalidad en cada caso, suministran información muy variada sobre las posibles líneas de causalidad y la intensidad de la interacción entre las distintas variables observadas.

Subraya Livi Bacci, como argumentos en contra de la supuesta relación lineal entre carestía y mortalidad, varias conclusiones que se derivan de ese escrutinio de las grandes crisis. En primer lugar, no fueron raros los casos de fuertes alzas de los precios de los alimentos sin consecuencias apreciables sobre la mortalidad; en alguna ocasión, incluso, como en la Toscana en 1709, la carestía está «precedida» por el alza de la mortalidad -y no al revés, como sugiere la interpretación clásica de las «crisis de subsistencias»- En segundo lugar, casi siempre es una explosión epidémica bastante bien definida la que puede hallarse «como causa directa» de grandes alzas de mortalidad, haciendo muy problemática su conexión con el nivel alimentario de la población cuando se trata de epidemias como, por ejemplo, la peste. Por último, incluso cuando la mortalidad responde de manera clara a las elevaciones de los precios, su reacción es muy desigual en cada caso, debido a múltiples razones que resulta prácticamente «imposible determinar» (pp. 89 y 91).

En lo que atañe a la tercera de estas observaciones, no parece que, de momento, se pueda decir más que la carestía, y sobre todo el alza de los precios, no es sino un indicador aproximado del estado real de nutrición,

\footnotetext{
${ }^{12}$ La cursiva es nuestra.
} 
y que, aunque fuera un indicador preciso, la variable alimentaria sólo explicaría parcialmente las variaciones de la mortalidad, dejando inexplicado un residuo, tal vez amplio, imputable a otra serie de factores. Es cierto, por otra parte, que hay grandes mortalidades que responden a epidemias «puras» -como es el caso frecuente de la peste o de la viruela-, y que es sumamente raro encontrar combinadas la carestía y la sobremortalidad sin rastro alguno de epidemia. Livi Bacci tiende a explicar este fenómeno por «la dislocación social» que rodea a la crisis de subsistencias, esto es, por la combinación de los factores que componen el «síndrome de miseria» o atraso - pobreza, suciedad, ignorancia, mendicidad ambulante...-, que asociados a la malnutrición se agravan en época de carestía y explican la incidencia y la letalidad de las epidemias subsiguientes tal vez mejor que el hambre propiamente dicha ${ }^{13}$.

Por supuesto, hay que reconocer validez explicativa a este razonamiento, un desarrollo del concepto de «hambre oculta» que, a su vez, ha sido definido por Carmichael como «la carga ambiental añadida a la pobreza y que junto a ella crea las circunstancias propicias para repetidas y múltiples infecciones» ${ }^{14}$. Creo, además, que esa referencia al marco social de las crisis es una de las indicaciones más útiles que el estudioso de estos fenómenos puede extraer de la lectura del libro de Livi Bacci, y proporciona por sí misma un rumbo nuevo a las investigaciones futuras sobre la mortalidad del pasado. Pero es plausible pensar que, en muchas ocasiones, una prolongada desnutrición podía favorecer la difusión de ciertas enfermedades y, sobre todo, agravar la letalidad de procesos de infección que hasta ese momento no habían manifestado caracteres epidémicos. Como también es cierto que la epidemia por sí sola podía ser, al igual que las grandes carestías, perturbadora del marco social y generadora de aquel «síndrome de miseria» que incluye entre sus ingredientes a la escasez y el hambre. Todo ello son vías diversas que, en cualquier caso, sirven para explicar esa frecuente coincidencia de enfermedad y carestía. Lo que tal vez no sea tan útil subrayar es el hecho de que, en última instancia, el agente directo de la sobremortalidad es finalmente la epidemia. Y ello porque, en situaciones concretas de extremada y prolongada malnutrición, o en un caso hipotético de "hambre sin epidemia» (del que sólo mirando con lupa puede encontrarse algún ejemplo en los anales de la historia), el hambre puede convertirse por sí sola en un fenómeno epidémico, con sus manifestaciones clínicas bien conocidas por los especialistas - y por la experiencia casi cotidiana de muchas regiones actuales del Tercer Mundo-, y al que se podrían atribuir, como causa específica de mortalidad, muchas de las víctimas de la crisis. Eso es, por otra parte, tal y como se nos ha recordado atrás (pp. 74-78),

${ }^{13}$ Véase el desarrollo de esta explicación, entre otras, en las pp. 59, 81 ó 91 del libro de Livi Bacci.

${ }^{14}$ Ann G. Carmichael (1985), p. 52. 
lo que sucedió en los casos de poblaciones hambrientas durante las dos guerras mundiales de nuestro siglo, en los que el hambre por sí misma fue el principal agente de mortalidad, con independencia de los procesos epidémicos, cuyos efectos relativos fueron secundarios.

Todas estas reflexiones, y alguna más, se desprenden de un examen atento de las conexiones entre carestía y sobremortalidad en el pasado, cuya correcta interpretación es, como se ve, bastante complicada, en parte porque durante mucho tiempo se ha atenido a un modelo explicativo de dudosa validez universal. Se trata de la teoría clásica de las crisis de subsistencias, expuesta por J. Meuvret en $1946{ }^{15}$, y cuya «cadena lógica» se recoge también en el libro que nos ocupa (p. 80). De forma aún más resumida, lo que dicha «teoría» nos muestra es una secuencia lineal de accidentes climáticos, quiebra de la producción agraria, escasez, carestía, empobrecimiento del consumo, morbilidad epidémica y elevación de la mortalidad por encima de su nivel ordinario. La interrupción de esa secuencia en cualquiera de sus fases, o una inversión en el orden en que se encuentran algunos de sus elementos, inevitablemente han de suscitar dudas respecto a la validez del modelo, y esto es precisamente lo que ocurre cuando nos encontramos con carestías «sin eco apreciable sobre la mortalidad», o con elevaciones de la mortalidad que preceden a las de los precios.

A propósito de una importante crisis española del siglo xviII, que refleja precisamente la última de las eventualidades que acabo de mencionar, expuse ya hace algún tiempo mis objeciones a la «teoría» clásica de Meuvret, y propuse una explicación provisional de carácter alternativo ${ }^{16}$. Hay que advertir que en el corto muestrario de crisis cuya evolución aparece dibujada en los gráficos de las páginas $85-87$ y $90-91$ del libro de Livi Bacci, no sólo es la sobremortalidad de 1709 en la Toscana la que precede a la carestía en la región. Ocurre otro tanto con los fallecimientos en Siena en 1554, cuya elevación, aunque prosigue en 1555 , sólo en esta última fecha se ve acompañada por el alza de los precios. Estos ejemplos, como el de la crisis española de 1786-1790, son más abundantes de lo que pudiera creerse, y tendrían su explicación si la «teoría clásica de las crisis de subsistencias» fuera debidamente reformulada.

Si las fuentes históricas nos permitieran cuantificar, y mostrar gráficamente, algunos de los elementos esenciales en la secuencia que describe dicha "teoría», sus limitaciones habrían sido denunciadas mucho antes. La morbilidad epidémica, concretamente, aparece, como es lógico, por delante del alza de la mortalidad, pero detrás de la carestía y como efecto derivado de esta

${ }^{15}$ Jean Meuvret (1946).

16 Vicente Pérez Moreda (1984). 
última, lo cual supone admitir como necesaria una línea causal cuya univocidad, por lo que ya hemos visto, no es tan lógica. Ocurre que los historiadores contamos con innumerable documentación cuantitativa referente a las defunciones, pero casi nadie conoce cifras acerca de la morbilidad, a no ser en casos muy excepcionales como el de la crisis española en 1786-87. Por eso, aunque sabemos que casi nadie se muere de repente y que la mayor parte de los fallecimientos tienen su origen en la morbilidad de semanas o meses atrás ${ }^{17}$, solemos dar por válido el orden causal entre carestía y mortalidad incluso cuando las cifras nos muestren una perfecta sincronía, o un mínimo lapso de tiempo, en la elevación de ambas variables. De forma que lo que es una simple secuencia temporal del tipo (a), o una simultaneidad del tipo (b), con la supuesta dirección de causalidad que sugiere el modelo, en realidad encubre una relación causal como la esquematizada en (c):

(a) carestia - ? $\rightarrow$ mortalidad

(b)

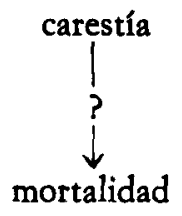

(c)

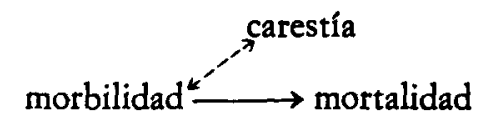

Así pues, en algunas ocasiones podría observarse una secuencia causal acorde con el modelo «clásico», a saber:

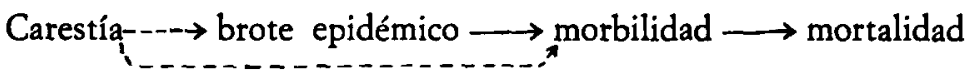

Pero, en otras, la secuencia sería la siguiente:

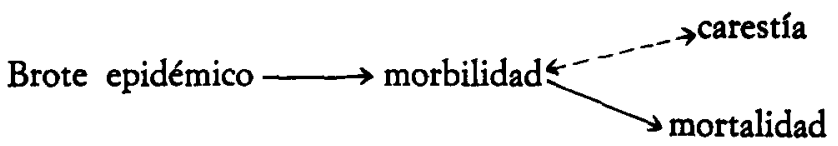

En todos los esquemas anteriores, por morbilidad y mortalidad se entiende siempre, claro está, morbilidad epidémica y sobremortalidad, y las líneas continuas indican relaciones necesarias, mientras que las discontinuas señalan sólo las relaciones contingentes. La relación entre epidemia y mortalidad es,

${ }^{17}$ Salvo en procesos epidémicos como el de la peste, donde el curso de la enfermedad y su desenlace fatal suele ser cuestión de días. 
efectivamente, necesaria, mientras que los vínculos entre carestía y mortalidad son simplemente eventuales $y$, cuando se producen, se manifiestan siempre a través de la vía epidémica. Así se explica que la frecuente coincidencia temporal entre el alza de precios y la de la mortalidad no implique siempre una necesaria relación de causalidad entre ambos fenómenos, o que la carestía, consecuencia a veces de la morbilidad epidémica y de la perturbación social que ésta origina, pueda aparecer simultáneamente, o incluso con posterioridad, a la crisis de mortalidad propiamente dicha.

Por lo dicho hasta ahora, resultará difícil compartir algunos de los juicios críticos acerca del análisis econométrico de las relaciones a corto plazo entre precios y mortalidad, emprendido por R. D. Lee y desarrollado por P. Galloway, T. Richards y D. Weir, entre otros ${ }^{18}$. Dice Livi Bacci que los resultados que se desprenden de esos análisis son «interesantes, pero contradictorios», y que «no carecen de ambigüedades interpretativas» (pp. 92 y 94). Requieren, ciertamente, una cuidadosa interpretación, del tipo de la que el mismo Livi hace en las páginas siguientes, pero las características del análisis y de los datos analizados - los de Inglaterra, concretamente- permiten eliminar cualquier ambigüedad o contradicción en los resultados.

En un amplio período como el que comúnmente contempla el análisis hay grandes elevaciones de los precios junto a otras menores, como también hay coyunturas en las que los precios apenas varían y otras en las que descienden. Es decir, el análisis no intenta medir la elasticidad de la mortalidad frente a la carestía, sino respecto a la variación de los precios. Si sólo un porcentaje bastante modesto de la varianza de la mortalidad es explicada por las variaciones de los precios es porque, como ya hemos visto, la carestía no es siempre la variable más importante -y mucho menos la única- que actúa sobre la mortalidad, y su relación causal con esta última ha de disminuir más aún cuando las variaciones de los precios, menores o de signo negativo, no permiten ya hablar de carestía. El «débil» poder explicativo de los precios sobre la mortalidad no es, pues, una limitación del modelo, sino uno de sus resultados «fuertes», puesto que es totalmente acorde con las expectativas teóricas. Tampoco debe sorprender que, a medida que avanza el tiempo, la relación entre precios y mortalidad, en Inglaterra al menos, se vaya debilitando, y que en el período de 1746-1834 llegue a ser incluso de signo negativo. Si Livi Bacci hubiera incluido en su tabla los niveles de significación de

" El pionero de este tipo de análisis fue Ronald Lee (1981), en su colaboración en la obra de E. A Wrigley y R. S. Schofield (1981). Importantes aplicaciones del análisis han (1983), David R. Weir (1984) y Patrick R. Galsido, entre otras, las de traducción de este último artículo, junto al análisis de datos españoles por parte de Vicente Pérez Moreda y David-Sven Reher, aparecerán en el Boletin de la $A D E H$, VI (1988), 3. 
los coeficientes estimados, podríamos ver que esa relación de signo negativo también es estadísticamente mucho menos significativa que en períodos anteriores ${ }^{19}$. Podríamos concluir, por tanto, que en ese período final la mortalidad es ya completamente indiferente a las variaciones de los precios. Por ello es acertado sugerir que, en el caso inglés, «a través del tiempo, habría una progresiva alteración de la relación precio/mortalidad, hecho que concuerda bien con la hipótesis de una neta mejora de la situación alimentaria en los siglos XVIII y xIx que haría poco sensible el estado de salud de la población a las variaciones del poder adquisitivo» (p. 94). No parece ésta una conclusión contradictoria. $\mathrm{O}$, mejor dicho: puede ser contradictoria con la argumentación de Livi Bacci, precisamente porque podría servir para apoyar alguna de las tesis de McKeown.

Los capítulos cuarto y quinto siguen agrupando pruebas en contra de la primacía del factor alimentario en la mortalidad y el crecimiento demográfico. No deja de sorprender la originalidad de la pesquisa y la contundencia de algunas de estas lecciones extraídas de la historia demográfica, económica y social, por más que la causa de la «hipótesis alimentaria», contra la que se intenta combatir, esté, como ya hemos visto, perdida de antemano. Las más importantes conclusiones que, tras una jugosa discusión, se deducen de estas páginas podrían ser resumidas como sigue.

La mortalidad de los grupos sociales más privilegiados, las élites en general, no fue significativamente inferior a la del resto de la población, a pesar de que tenían siempre garantizados unos niveles de alimentación suficientes y gozaban de un tenor de vida superior. La abundencia y riqueza de recursos alimentarios de los «nuevos» países americanos - Canadá, Estados Unidos, Argentina... - no generaron entre sus pobladores de origen europeo unos niveles de esperanza de vida sensiblemente superiores a los de algunas naciones de Europa por las mismas fechas. Los grandes contrastes que presenta, en el antiguo régimen demográfico, la mortalidad durante el primer año de vida estaban presumiblemente desvinculados de los factores alimentarios, dado el carácter casi universal de la lactancia materna, practicada por doquier en esas épocas, y deben ponerse más bien en relación con otros factores ambientales, como el clima, las prácticas de crianza, la actividad laboral de la madre o el grado de atención que se prestaba al niño. La estatura, como índice «neto» del nivel de nutrición, conoce un estancamiento en diversas zonas de Europa en las últimas décadas del siglo xviII y las primeras del XIX, paralelo al deterioro en el nivel de vida y en la calidad de la dieta de las masas populares, todo ello a pesar del claro declive de la mortalidad en algunos de esos paises europeos a partir del siglo xviII.

\footnotetext{
19. Ree (1981), p. 375.
} 
Alguna de estas pruebas, como la relacionada con las diferencias que exhibe la mortalidad infantil, resulta bastante convincente, en la forma en que está razonada y defendida. Otras, sin embargo, presentan puntos más débiles o añaden menos fuerza a la argumentación. El mismo autor reconoce que la hipotética mortalidad diferencial más ventajosa en los «nuevos» países americanos «debe esperarse sólo si se abraza la tesis que ve en la alimentación el factor principalísimo del nivel de mortalidad» (p. 110). Pero ya hemos visto lo difícil que resulta abrazar una tesis enunciada en tales términos. También los datos que se aportan al respecto merecerían, tal vez, algún matiz. A pesar de la crítica que se ha hecho a las estimaciones de Fogel sobre la esperanza de vida norteamericana a mediados del siglo XIX, habrá que reconocer que los niveles de mortalidad de la población blanca eran allí ciertamente inferiores a los de los esclavos ${ }^{20}$. Y no eran tantos los países europeos que hacia 1850 gozaban de una esperanza de vida al nacimiento de más de cuarenta años, como la de la población blanca de los Estados Unidos; mientras que eran muchos más aquellos en los que este índice de supervivencia era incluso bastante inferior al de la población esclava norteamericana.

Desde luego, las pruebas acumuladas a propósito de la mortalidad diferencial de los países de nueva colonización o de las élites privilegiadas «no son decisivas» (p. 112). Estas últimas podrían tal vez haber sido contrastadas con la referencia a otros estudios que muestran ventajas ligeramente mayores en la esperanza de vida de alguno de los grupos sociales dominantes, al menos en el siglo xvil ${ }^{21}$. Por otra parte, el argumento de que la aristocracia, sin tener que mejorar una dieta que siempre había sido buena, conoció también una sensible mejora de la mortalidad en el siglo xviII, reproduce exactamente la crítica ya antigua de Peter Razzell a los primeros enunciados de la tesis de McKeown, Brown y Record, y podría ser contestado, como ya se hizo hace tiempo, sin abandonar la defensa de las mejoras en la alimentación: si estas mejoras afectaron sólo a las masas populares y eso ayudó a reducir la circulación epidémica en el conjunto de la población, las clases superiores también se habrían visto indirectamente beneficiadas, a través de una menor exposición al contagio 22 .

Tampoco están libres de toda sospecha los argumentos empleados en la utilización de la talla como indicador del nivel de nutrición. Livi Bacci admite que no todas las piezas de la demostración encajan perfectamente y que el

${ }^{20}$ Robert W. Fogel y Stanley L. Engerman (1981), pp. 106-109. La esperanza de vida de los esclavos era, hacia 1850 , un 12 por 100 más baja que la media de los blancos norteamericanos.

${ }^{21}$ Véanse, por ejemplo, los datos de Dante E. Zanetti (1972), pp. 219-231; si bien la técnica empleada en la obtención de esos resultados no parece excesivamente rigurosa.

22 La respuesta al comentario de P. Razzell (1974) fue hecha por R. E. Quinault (1976) y por él mismo, Th. McKeown (1976), pp. 139-142. 
debate sobre el tema está «erizado de dificultades técnicas», que prefiere pasar por alto (p. 166). No encaja, efectivamente, la afirmación sobre el supuesto declive de la estatura de los reclutas suecos «hacia finales del siglo xvin»" (p. 167), puesto que los datos de la columna respectiva en el cuadro de la página siguiente revelan más bien lo contrario. Sí que desciende, sin embargo, a finales de ese siglo o en los primeros años del siguiente, la talla de los reclutas en las distintas zonas centroeuropeas de la monarquía de los Habsburgo, como muestran los restantes datos de ese cuadro. Al parecer, dicha talla conoce "una recuperación al término de las guerras napoleónicas» (p. 168). Uno se pregunta si no habrían sido rebajados los mínimos de talla exigibles en el reclutamiento a medida que aumentaban los conflictos bélicos con el fin de ampliar las levas, tal y como ha sucedido frecuentemente en estos casos. De cualquier forma, el simple aumento del contingente militar por una incrementada demanda bélica es probable que condujera a una menor selectividad de los reclutas y a una disminución de su talla media. Esta es una de las muchas dificultades técnicas con que se enfrenta la interpretación de los datos históricos referidos a la estatura.

Es interesante comprobar que la experiencia histórica demuestra que las poblaciones europeas gozaban, por lo general, de una dieta media superior a las 2.000 calorías diarias per capita, suficiente, como se ha demostrado más arriba, para evitar el riesgo de mortalidad que hubiera supuesto descender por debajo de ese umbral. Ello nos conduce, claro está, a restringir el examen de las posibles conexiones entre desnutrición y mortalidad a la coyuntura catastrófica, al corto plazo de las más dramáticas carestías. Pero no significa que podamos retirar una importante cuota de protagonismo al hambre en la evolución de la mortalidad e incluso del crecimiento demográfico europeo: todo depende de la frecuencia y la intensidad que registraran aquellas breves etapas de coyuntura catastrófica. En este contexto tendrán que ser evaluados juicios como los que se emiten en estas páginas. Afirmar, por ejemplo, que «la agricultura europea debiera ser absuelta de la acusación de haber provocado el hambre a las poblaciones, al menos en épocas indemnes de catástrofes" (p. 140), equivale más o menos a decir que no hubo hambre... excepto cuando sí la hubo; y asegurar que «una base calórica suficiente debía alcanzarse con facilidad en tiempos normales y para quien viviera en condiciones no marginales» (p. 146) implica que era más difícil lograrla cuando corrían tiempos excepcionales $o$, en cualquier caso, entre la población marginal, siempre mucho más numerosa en el pasado que en las sociedades desarrolladas de nuestros días.

La discusión de los posibles factores del declive de la mortalidad en el siglo xviII (pp. 113-117), y de la relación que al respecto pudieron guardar los nuevos cultivos y la posible mejora de la alimentación en el período, es ambigua y poco concluyente. Livi Bacci se muestra partidario de una inter- 
pretación plural del proceso -en el que debieron de intervenir, junto a las mejoras en la alimentación, los avances en la higiene o en la medicina, así como los cambios en la virulencia de ciertas enfermedades o en las resistencias inmunitarias frente a ellas-, sin pasar a pronunciarse sobre la importancia relativa de cada uno de estos factores individuales. Creo que esta interpretación, como hipótesis de trabajo, es más admisible que la explicación monocausal de McKeown, basada en la primacía exclusiva de las mejoras alimentarias. Pero para convertirse en una tesis totalmente convincente debería ser más y mejor argumentada. Sigue siendo muy dudoso, por ejemplo, que la medicina del siglo xviII contribuyera «ciertamente a disminuir la incidencia de la viruela» (p. 117), a pesar de los vehementes intentos de P. Razzell por convencernos de lo contrario ${ }^{23}$.

En cuanto a los progresos en la nutrición a lo largo del siglo xvirI, que es donde se centra la polémica con la tesis de McKeown, subsiste en Livi Bacci cierta contradicción cuando admite, por un lado, «los positivos efectos de las aumentadas disponibilidades» alimentarias y, poco después, pasa a dudar de "la existencia incluso de mejoras de la alimentación anteriores al siglo xIX" (pp. 151 y 154). Ya se advirtió más arriba que no conviene confundir posibles deterioros en la calidad de la dieta y en el nivel de vida en general, visibles a través de ciertos indicadores en las fechas que rodean a 1800 , con aumentos en la oferta de subsistencias, que, al parecer, nadie pone en duda y que debieron de impulsar el crecimiento demográfico del período. Defender que esta conexión entre el aumento de la oferta alimentaria -propiciado por la extensión general de las roturaciones, la introducción de cultivos nuevos, la mejora de los transportes y la mayor regularidad del suministro- y el crecimiento demográfico se ejerciera exclusiva o básicamente a través de la caída de la mortalidad, sin tener en cuenta la intervención de la nupcialidad y la fecundidad en el proceso, es, sin duda, el punto débil de la tesis de McKeown. Pero también la mortalidad, incluso en Inglaterra, se pudo beneficiar de todas esas mejoras, especialmente las que afectaron a las comunicaciones y a las técnicas de distribución, que condujeron, ya desde finales del siglo xvir y principios del xvirI, a una progresiva integración de los mercados y permitieron una mayor especialización y diversificación de la producción, lo que

${ }^{23}$ Las tesis sobre los efectos de la inoculación y de las primeras vacunaciones, defendidas por Peter Razzell (1976 y 1977), han recibido muy duras críticas por parte de los especialistas. Véanse, como prueba de ellas, las reseñas respectivas de J. M. LANE, en The Journal of Economic History, 37 (1977), 4, pp. 1086-1087, y de G. Gronje, en The Economic History Review, 31 (1978), 4, pp. 665-666. Tampoco resultan convincentes las pruebas que aporta, en rélación con la eficacia de la inoculación en la lucha contra la viruela en el siglo xvirI, A. J. Mercer (1985). 
habria hecho disminuir, al menos, la frecuencia y el impacto de las carestías locales ${ }^{24}$.

La polémica sobre el papel de la alimentación en el crecimiento del siglo xviII, aunque se va clarificando, sigue, por lo tanto, aún sin resolver. Es lamentable que las dos tesis que se enfrentan con el tema concluyan en demostraciones a contrario, por vía de exclusión de las restantes explicaciones alternativas. McKeown afirma la primacía del factor alimentario porque cree insuficientemente fundados los argumentos que recaen sobre los demás factores que pueden haber influido en el descenso de la mortalidad, pero no aporta evidencias concretas sobre los progresos en la nutrición. Como reconoce el mismo Livi Bacci, la tesis de McKeown «tiene probablemente un núcleo de verdad, pero necesita ulteriores pruebas» (p. 152). La tesis de Livi Bacci, por su parte, también las necesita, porque defiende que la alimentación no fue el único ni el principal factor en el declive de la mortalidad, pero rehúye el examen individualizado de los demás factores posibles. No es poco que esta obra sirva para remitirnos de nuevo al problema, pero es preciso reconocer que no lo resuelve.

He dejado para el final una observación escéptica sobre el empleo que Livi Bacci hace de los salarios reales, y de su «sorprendente» evolución paralela a la de la mortalidad. El mismo autor inicia esta sección de la obra con juicios muy escépticos acerca de la capacidad de los salarios reales para afrontar el problema, y la concluye afirmando que un análisis más detallado de las conexiones entre salarios reales y mortalidad «no daría más fuerza al tema» (p. 165). Estoy totalmente de acuerdo con lo primero, y también con lo segundo, porque una inspección teórica de la naturaleza de esa conexión no sirve, en mi opinión, para apoyar mejor los argumentos que se exponen: más bien, elimina el poder explicativo que pretende tener el razonamiento.

Este razonamiento es, en suma, el siguiente: si los salarios reales son un indicador del nivel de vida de la población, y concretamente de su nivel de alimentación, y si esta última tiene algo que ver con la mortalidad, tendríamos que esperar una conexión positiva entre salarios reales y esperanza de vida. Pero lo que las series disponibles de estos indicadores nos muestran es más bien lo contrario: al menos hasta bien entrado el siglo xIX, se aprecia una evolución divergente entre los ciclos del índice de supervivencia y los de los salarios reales. Es decir, la mortalidad aumenta cuando el nivel de vida (el salario real) sube, como en los últimos siglos medievales o durante buena parte del siglo xviI, y desciende cuando los salarios reales están en retroceso, como ocurre, al parecer, en el siglo xvi y en la segunda mitad del siglo xviII.

24 Esto es lo que sugiere R. Lee (1981), p. 376. Y es la interpretación que defiende R. S. Schofield (1985), pp. 91-92. 
No son pocas las limitaciones con que cuentan los indicadores disponibles del salario real cuando se pretende utilizarlos como medida del nivel del poder adquisitivo y, más aún, del nivel de alimentación del pasado. En muchas de ellas se detiene Livi Bacci, y merece la pena recordar algunas. El salario real es un cociente entre el salario nominal o monetario y un índice de precios (de los cereales u otro indice de precios al consumo), pero suele reflejar sólo la evolución del poder adquisitivo de un sector muy minoritario de asalariados, por lo general del artesanado urbano y, frecuentemente, de los obreros del sector de la construcción. Ni siquiera puede medir bien la capacidad de compra de las remuneraciones totales que estos grupos de asalariados recibían, porque en buena parte del período considerado el salario monetario puro era una rareza, siendo lo más frecuente que las remuneraciones íntegras fuesen de naturaleza mixta, incluyendo también pagos en especie. Por otra parte, el campesinado, que constituía la mayor parte de la población durante épocas preindustriales, así como los artesanos y muchos de los habitantes de las ciudades, se abastecían parcial o totalmente por vía del autoconsumo, a través de recursos alimentarios asequibles fuera de los circuitos del mercado. No insistiré demasiado sobre este fenómeno del autoconsumo y su posible grado de extensión —variable según épocas, países y sectores sociales-, entre otras cosas porque sus efectos también debían de quedar registrados en el mercado, a través del sistema de precios, y deberían acusarse, por tanto, en los salarios reales.

Lo que sí resulta importante es subrayar que los campesinos eran productores de alimentos, y «no está claro qué podía significar para los productores un desgaste de los salarios reales" (p. 157). Cuando el indicador del salario real está fuertemente afectado por el precio de los alimentos básicos, y si exceptuamos una hipotética situación en la que la mayor parte de la producción agraria se destinara exclusivamente al autoconsumo (situación que en nada se parece, por supuesto, a la de muchos países de la Europa occidental en esa época, y menos aún a la experiencia inglesa del siglo xviri, a la que se presta especial atención en esas páginas), parece claro que una tendencia decreciente del salario real había de ser positiva para los campesinos, en la medida que implicaba un aumento relativo de los precios de venta de sus excedentes agrarios sobre la remuneración monetaria del trabajo.

Hay que tener en cuenta, por fin, que el salario real puede ser un índice del poder adquisitivo o de la capacidad de consumo, pero nunca de la demanda real de productos alimenticios - a no ser en el caso extremo de unos salarios de estricta subsistencia o de muy violentas alzas coyunturales de los precios-. Pues si el nivel medio de los ingresos reales supera el exigido por el umbral de subsistencia, siempre queda un margen, mayor o menor, para el consumo de otros bienes que no sean los alimentos populares o de primerísi- 
ma necesidad. En esta situación, incluso el asalariado puede «soportar» una elevación, tal vez importante, de los precios de los alimentos básicos, y una caída consiguiente del salario real, sin que descienda su nivel ordinario de alimentación. La mayor rigidez de la demanda de esos productos de consumo inexcusable hará que cada vez se gaste una mayor proporción de los ingresos salariales en ese capítulo, en detrimento de otros bienes de demanda más elástica y cuyo consumo se puede fácilmente sustituir o aplazar. Por eso es muy cierto que, por encima de la tendencia que adopten los salarios reales, interesa observar si sus niveles son suficientes para garantizar un adecuado nivel de alimentación (p. 164). La consideración de este efecto de las variaciones en la renta sobre la demanda de bienes elásticos e inelásticos puede abrir importantes vías al tema que nos ocupa: es muy probable, por ejemplo, que muchas elevaciones de los precios de los alimentos en el pasado no tuvieran repercusiones notables sobre el consumo de ese tipo de productos, y sí implicaran, por el contrario, una importante reducción de la demanda de otro tipo de bienes. También cabe pensar que una caída del consumo de algunos de estos otros bienes considerados de menor necesidad -como vestido, calefacción o medicamentos- podría por sí misma provocar o agudizar ciertos procesos de morbilidad y hacer aumentar el número de defunciones, sin que la situación llegara a provocar el hambre, como se ve, aun estando asociada a una coyuntura de típica carestía.

Son muchas, pues, las precauciones que hay que tomar cuando se examina el significado de los salarios reales, y es muy precipitado intentar extraer conclusiones rápidas del simple paralelismo de las tendencias o los ciclos del salario real y de la mortalidad. La posible conexión entre ambos fenómenos debe buscarse, además, en el corto plazo principalmente, en el que apenas fluctúan los salarios nominales y donde toda variación importante en el nivel de vida debe quedar registrada en la fluctuación de los precios. Eso es lo que hizo, como vimos, R. Lee al analizar el caso inglés, encontrando, por cierto, que en el período de aproximadamente 1750 a 1830 las fluctuaciones de los precios de los cereales no influyeron para nada en las de la mortalidad, a pesar de esa aparente coincidencia tendencial en el ciclo 'de la mortalidad y de los salarios reales. Por otra parte, la construcción misma del salario real y su representatividad están lejos de ofrecer garantías plenas al historiador. Esta misma observación se puede hacer, por supuesto, cuando pensamos en los datos ingleses, con referencia a la gran obra de Wrigley y Schofield cuyas series se utilizan aquí. Pero no seré yo quien la haga, porque ya la hizo un buen conocedor de ese trabajo y de la historia de su país: su colega en el grupo de Cambridge, Peter Laslett, quien prefiere limitarse a examinar las relaciones entre la mortalidad y los precios del trigo - como hace Lee- y no extenderse, como hacen «exhaustivamente" Wrigley y Schofield en su obra, 
en las conexiones entre los salarios reales y las distintas variables demográficas, «sobre todo porque la ubicación, en el sur, de la serie de salarios, y su estrecha base en [el sector de] la construcción, cäusa mucha incertidumbrew ${ }^{25}$.

Si no fueran suficientes estas observaciones acerca del limitado poder explicativo del salario real en el contexto de los problemas que discute la obra de Livi Bacci, merecería aún la pena reflexionar algo más sobre su misma naturaleza y sus posibles determinantes. Porque el «salario real» no es un dato histórico, un fenómeno del pasado, como sí lo eran la mortalidad o los precios. Es, por el contrario, un concepto inventado por los economistas, que se construye a partir de datos históricos como son los salarios monetarios y los precios y que emplean a menudo los historiadores para acercarse lo mejor posible, tal vez sólo de lejos, a lo que podría ser el nivel de vida de los asalariados. Es un producto que depende de los elementos que intervienen en su construcción y de las fuerzas que actúan sobre cada uno de estos elementos.

Esta perorata, aparentemente innecesaria, viene a cuento porque la argumentación de Livi Bacci induce a pensar en el salario real como si fuera una variable exógena, de la que dependen, o podrían depender, el nivel de nutrición $y$, tal vez, la mortalidad. Por el contrario, el salario real es una variable dependiente, determinada por la evolución de los incrementos relativos de precios y salarios nominales $y$, en última instancia, de los factores que in. fluían en estas dos variables económicas. Entre estos factores destacan el volumen de población y la tasa de crecimiento demográfico, que, a través de sus efectos sobre la oferta de trabajo y la demanda de productos finales, influyen, respectivamente, en salarios y precios. Ahora bien, el crecimiento demográfico estaba determinado, aunque no exclusivamente y tampoco siempre de manera principal, por los niveles y las tendencias de la mortalidad. De modo que la mortalidad y su tendencia, si se quiere por vía indirecta, determinaban parcialmente al menos los niveles del salario real en cada momento, y no viceversa. Y si en muchas ocasiones la mortalidad era un elemento exógeno en el sistema demográfico -y esto es lo que subraya acertadamente el libro de Livi Bacci-, también era un elemento exógeno en el sistema económico por medio de esa influencia sobre precios y salarios a través del crecimiento demográfico. En la compleja ecuación que define los elementos de los que es función el salario real tendría que figurar la mortalidad, y de ahí procede la coincidencia tendencial que podemos observar en muchos períodos del pasado entre los indicadores de ambas variables. Deberíamos explicarla, por tanto, diciendo que en economías muy inerciales y cuya capacidad productiva tarda en adaptarse a las bruscas oscilaciones de la población, el ascenso de los

${ }^{2 s}$ Peter Laslett (1983), p. 178, n. 24. Recuérdese que el análisis de Lee se incluye, como ya se ha dicho, en el cap. 9 de la obra de Wrigley y Schofield (1981). 
salarios reales es una inevitable consecuencia de épocas de mortalidad especialmente acusada, como la Baja Edad Media o el siglo xvII; y su descenso, como el que se advierte a lo largo del siglo xvi o en la segunda mitad del xviII, es un efecto principalmente del crecimiento demográfico, propiciado, aunque tal vez de forma no exclusiva, por el aumento de la esperanza de vida ${ }^{26}$. El paralelismo entre ambas tendencias no es una «sorprendente» paradoja, sino un fenómeno del todo acorde con las expectativas teóricas, que el historiador comprueba universalmente, en épocas anteriores al siglo xIx, y que tiene caracteres inevitables por la misma naturaleza del concepto de salario real.

Interesa subrayar que todas las observaciones críticas anteriores afectan a la calidad o el peso específico de algunas de las pruebas que Livi Bacci aporta para defender una tesis que en lo esencial comparto, como lo hacen hoy día buen número de especialistas: el papel muy relativo, y quizá sólo ocasional en el tiempo y minoritario en el conjunto del cuerpo social, de la desnutrición en la mortalidad y el crecimiento demográfico del pasado. Al fin y al cabo, se nos viene a decir, la influencia negativa de la carestía sobre la población está fuera de toda duda, aunque las mayores crisis de mortalidad estuvieran provocadas por el factor epidémico y su vínculo con el factor alimentario fuera menor o inexistente. La sobremortalidad que originaron todas esas crisis constituyó un freno importante, y su atenuación, que en el siglo xviII se logró «con la introducción de nuevos cultivos, el desarrollo y la integración de los mercados o la difusión de medidas de ayuda» (p. 186), contribuyó a impulsar el moderno crecimiento de la población. Pero, en intermitentes oleadas, ese freno correctivo malthusiano había actuado por medio de la escasez de los recursos alimentarios. Obviamente, la alimentación debe ser «absuelta de la imputación de haber constituido el elemento determinante de la mortalidad en años de normalidad», pero "al menos en parte es culpable de haber frenado el crecimiento con más o menos frecuentes años de penuria” (p. 187). Así, pues, la influencia de la nutrición $\multimap$ del hambre - debe ser tenida en cuenta entre una pluralidad de factores que explican las diferencias y las variaciones de la mortalidad del pasado: «negar que tuviese algún efecto sobre el grado de mortalidad sería pueril. Afirmar que ejerce un efecto decisivo resulta infundado" (p. 112).

Este libro tiene la valentía de enfrentarse con un tema bastante complicado, por su carácter interdisciplinario y porque probablemente choca con las creencias rutinarias de quienes todavía interpretan con excesiva precipitación las posibles o aparentes relaciones entre el hambre y la mortalidad. Invita, por otro lado, a desarrollar más el análisis detenido de estas conexiones, tra-

${ }^{26}$ Los datos ingleses, al menos, documentan bien este aumento de la esperanza de vida en la segunda mitad del siglo XVI y, por supuesto, a lo largo del siglo xviII. 
tando de aislar los factores que constituían el «síndrome de miseria» y que intervenían, junto con la desnutrición, en muchas situaciones de sobremortalidad. Supone, por todo ello, un importante estímulo a las investigaciones sobre la mortalidad, relativamente rezagadas en los últimos tiempos en relación con los desarrollos que ha registrado el análisis de otros sectores de la demografía, como la fecundidad o la nupcialidad. Es de lamentar, por todo ello, que no se haya cuidado precisamente la edición en castellano de una obra que aparece casi simultáneamente traducida en varios idiomas y que va a ser objeto de especial atención durante algún tiempo en muchas latitudes. Estas páginas han tratado de responder al desafío intelectual que la obra plantea, y las que siguen intentan aliviar, en la medida de lo posible, ese descuido editorial.

\section{APENDICE \\ Orientaciones para el lector de la traducción española}

Es usual, en notas bibliográficas como ésta, señalar, mediante unos cuantos ejemplos concretos, la presencia de alguna errata o de algún fallo en la traducción, siempre con el loable objetivo de advertir a los editores acerca del cuidado que deberían prestar a la calidad final de su producto. En esta ocasión, la finalidad de la «fe de erratas» que sigue no es sólo ésa, sino principalmente la orientación del lector. Pues es tal la cantidad de tales «erratas», y sobre todo de deficiencias en la traducción del texto al castellano, que el lector medio difícilmente habrá podido enterarse del pensamiento del autor en muchos pasajes de la obra, y es posible que un lector más familiarizado con el tema haya optado, indignado, por cortar la lectura mucho antes de llegar a la última página del libro. Así, pues, a modo de crítica, severa pero constructiva, figura esta relación de los principales errores detectados, que ha sido realizada cotejando cuidadosamente el texto de la versión española con el del manuscrito italiano.

Pág. 9

(Líneas 32 y 34): Donde dice «la significativa de los principales indicios... es imprecisa y discutidaw, debería decir «el significado [o, en el peor caso, la significatividad] de los principales indicios... es impreciso(a) y discutiblew.

Pág. 15

(Líneas 11 y 12): Donde dice «de los siglos xIII al xv o del XVI», debería decir «de los siglos XIII, XV o XVI*. 
Pág. 18

(Línea 39): Donde dice «uniformidad de población», debería decir «uniformidad de la densidad».

Pág. 20

(Línea 39): Donde dice «contando los hijos*, debería decir «contando las hijas».

Pág. 21

(Líneas 8 y 9): Donce dice «estacionalidad», debe decir «estancamiento».

(Líneas 15-16): Donde dice «tasa bruta de reproductividad», debería decir «tasa bruta de reproducción».

(Línea 24): Donde dice «hijos por mujer», debe decir «hijas por mujer».

Pág. 22

(Líneas 20 y 23): Donde dice «expectativa(s) de vida», debería decir «esperanza(s) de vidaw.

(Línea 21): Donde dice ahijos por mujer ( $R$, eje de las coordenadas)», debe decir ahijas por mujer $(R$, eje de las ordenadas)

(Línea 28): Donde dice «reproductividad», debería decir «reproducción bruta».

Pág. 23 (Gráfico 3)

En el título del gráfico, donde dice «Relaciones entre reproductividad, R», debería decir «Relaciones entre reproducción bruta, R».

En la leyenda del eje de ordenadas, donde dice «Niños por mujer», debería decir "Niñas por mujer».

$Y$, debajo del gráfico, los signos que indican los diferentes niveles de crecimiento deberían ir precedidos de $\alpha r=m, 0$ *Incremento $(r)=\$$.

Pág. 24

(Línea 6): Donde dice «25 años», debe decir «22 años».

(Línea 32): Donde dice «reproductividad», debe decir «reproducción».

(Línea 39): Donde dice «por lo tanto edad para el matrimonio», podría haber dicho «por lo tanto por la edad al casarse» (o «por la edad de acceso al matrimoniom).

(Línea 40): Donde dice «nubilidad definitiva», debe decir «soltería definitiva».

Pág. 25

(Líneas 5.6 y 9): Donde dice «reproductividad», debe decir «reproducción».

(Líneas 10 y 11): Donde dice *que pueden haber tenido en las modificaciones», debe decir *que pueden haber tenido las modificaciones».

Pág. 28 (Gráfico 4)

En la parte izquierda del gráfico (fase de «Expansión»), donde dice, en el recuadro inferior, «Nupcialidad en aumento», debe decir «Mortalidad en aumento».

Pág. 29

(Líneas 31 y 32): Donde dice «y la segunda inmediata*, debe decir «y la segunda mediata* (o *indirecta*).

Pág. 31

(Línea 40): Donde dice «la familia cepo», debe decir «la familia troncal».

Pág. 33

(Líneas 14 y 15): Donde dice «a finales del siglo xvirI y en la primera mitad del siglo XIX», debe decir «a fínales del siglo XIII y en la primera mitad del siglo XIV». (Línea 16): Donde dice «enlentecimiento demográfico», debería decir cualquier cosa menos eso: «menor crecimiento demográfico" o «crecimiento demográfico más lento», por ejemplo.

Pág. 35

(Línea 26): Donde dice «el riesgo de muerte si estos umbrales», debe decir «el riesgo de muerte, si estos umbrales».

Pág. 39

(Líneas 22 y 23): Donde dice ael efecto del enlentecimiento de las crisis», debería decir wel efecto de la disminución de las crisis». 
Pág. 41

(Líneas 14 y 15): Donde dice «En la paridad de $R_{0}$, debe decir «permaneciendo $R_{0}$ invariablew.

(Líneas 16 y 17): Donde dice «la tasa neta de reproducción, $R$, que no es sino la suma de los $f_{x}$ y que expresa», debe decir «la tasa bruta de reproducción, $R$, no es sino la suma de las $f_{x}$, y expresaw.

Pág. 42

(Línea 14): Donde dice "Wrigler», debe decir «Wrigley».

Pág. 46

(Línea 14): Donde dice «2.700 y 2.500 calorías», debe decir $« 2.700$ y 3.500 calorías».

Pág. 57

(Línea 12): Donde dice «contrastar», debe decir «contrarrestar».

Pág. 60

(Línea 33): Donde dice «o menos violenta», debe decir *o muy violenta».

Pág. 62

(Línea 17): Donde dice «antagónico y más bien sinérgico», debe decir «antagónico y no sinérgico» (o «antagónico antes que sinérgico»).

Pág. 63

(Líneas 15 y 16): Donde dice «agrave la curación del tifus», debe decir «agrave los efectos [o el desarrollo] del tifus».

Pág. 64

(Línea 10): Donde dice «y no poco», debe decir «un poco».

Pág. 71

(Líneas 25 y 26): Donde dice «estableciéndose», debe decir «estabilizándose».

Pág. 73

(Línea 12): Donde dice «Key», debe decir «Keys».

Pág. 80

(Línea 40): Donde dice «sin embargo», debe decir «en cualquier caso».

Pág. 82

(Línea 16): Donde dice «del larga», debería decir «a largo plazo».

Pág. 83

(Línea 4): Donde dice «tienen reflejos», debe decir «tiene efectos».

Pág. 84

(Línea 13): Donde dice «1527-1571», debe decir «1527-1534 y 1570-1571».

(Línea 16): Donde dice $\$ 1648-1641 \%$, debe decir $« 1648-1651 \%$.

(Línea 19): Donde dice «refleja a Francia», debe decir «sigue a Francia» (o «repite la cronología de Franciax).

Pág. 91

(Líneas 6 y 7): Donde dice «o bien por una diferente», debe decir *o bien una diferente».

Pág. 92

(Línea 32): Donde dice «variante», debe decir «varianza».

Pág. 101

(Líneas 37 y 38): Donde dice «F. Lebrynm, "Les crises de démographie en France" \#, debe decir $\alpha \mathrm{F}$. Lebrun, "Les crises démographiques en France" $"$.

Pág. 106

(Línea 26): Donde dice «y no puede dejar dew, debe decir «no puede dejar de».

Pág. 109

(Línea 17): Donde dice «esperanza de vida calculada en 20 años*, debe decir *esperanza de vida a los 20 años».

(Línea 19): Donde dice «a cuadros tipo basados», debe decir «según tablas tipo basadas*. 
Pág. 110

(Línea 28): Donde dice *en dirección de la ciudad», debe decir «en dirección a la ciudad».

(Línea 37): Donde dice *contrastar», debe decir «contrarrestar».

Pág. 114

(Línea 3): Donde dice «de 20 años más alta», debe decir «20 años más alta» (o «de 20 años más»).

Pág. 115

(Línea 3): donde dice «infecciosas», debe decir «infeccioso».

Pág. 120

(Líneas 5, 6, 14 y 37): Falta la llamada de la nota 35 (después de «muy esparcida»), y eso hace cambiar correlativamente la numeración de las notas siguientes de la página, de la 35 a la 39 , que son en realidad las notas 36 a 40 . La llamada de la nota 40 , en la página siguiente, debe ser suprimida.

Pág. 124

(Línea 11): Donde dice *era imputable*, debe decir *eran imputables».

Pág. 125

(Líneas 32 y 33): Donde dice «Un cuadro de mortalidad», debe decir «Una tabla de mortalidad».

Pág. 126

(Línea 19): Donde dice «del cuadrow, debe decir «de la tabla».

(Línea 21): Donde dice "cuadros», debe decir «tablas».

Pág. 128

(Líneas 1, 4 y 16): Donde dice «Vogel», debe decir «Fogel».

(Línea 31): Donde dice «los cuadros», debe decir «las tablas».

Pág. 129

(Linea 10): Donde dice «en la medida», debe decir «en la mediaw.

(Línea 40): Donde dice "Wriglet», debe decir «Wrigley».

Pág. 135

(Línea 37): Donde dice «derechos a ellas», debe decir «derecho a ellas».

Pág. 138

(Línea 4): Donde dice "cuadro 14», debe decir «cuadro 15\%.

Pág. 139

(Línea 11): Donde dice $«(1800-1900) »$, debe decir $«(1880-1900) »$.

Pág. 146

(Líneas 35 y 36): Donde dice «hasta el avanzado siglo xvI», debe decir «hasta bien avanzado el siglo XVI».

Pág. 147

(Línea 13): Donde dice «inextensible», debe decir «inelástica» (o «rígida»).

Pág. 148

(Línea 14): Donde dice «de período largo», debería decir «a largo plazo».

Pág. 150

(Líneas 9 y 10): Donde dice «en particular la patata en Europa central y septentrional», debe decir «en particular el de la patata en la Europa central y septentrional y el del maíz en la Europa meridional».

Pág. 152

(Línea 23): Donde dice «y dificultad», debe decir «y menores dificultades».

Pág. 153

(Línea 6): Donde dice «se producen mejoras», debe decir «no se producen mejoras». 
Pág. 155

(Línea 21): Donde dice «en las construcciones», debe decir «en la construcción».

Pág. 156

(Líneas 1 y 2): Donde dice «también para estas categorías los salarios reales no representaban», debe decir "tampoco para estas categorías los salarios reales representaban».

(Líneas 5 y 6): Donde dice «las integraciones en especies», debe decir «las aportaciones en especie».

(Líneas 6 y 7): Donde dice «las integraciones en el balance monetario», debe decir "las aportaciones al presupuesto monetario».

(Línea 19): Donde dice «las parejas», debe decir «los hogares».

(Líneas 20 y 21): Donde dice "permitido de los jardines", debe decir «que posibilitaban los jardines".

(Línea 37): Donde dice «medieros», mejor sería haber dicho «aparceros».

Pág. 157

(Líneas 4 y 5): Donde dice «Carestía y alto costo es miseria. Abundancia y alto costo es opulencia», debe decir «Escasez y carestía es miseria. Abundancia y carestía es opulencia».

Pág. 162

(Línea 11): Donde dice «en nivel asociado», debe decir «a nivel agregado».

Pág. 163

(Línea 11): Donde dice «En el mismo gráfico 20», debe decir «En el gráfico 20».

Pág. 166

(Línea 25): Donde dice "que se refleja en el efecto "neto"», debe decir "cuyo efecto "neto" registra".

(Líneas 26, 27 y 28): Donde dice «lo neto de los efectos negativos tienen sobre la utilización de los principios nutritivos para el crecimiento la intensidad...», debe decir «una vez eliminados los efectos negativos que sobre la utilización de los principios nutritivos para el crecimiento tienen la intensidad.....

Pág. 167

(Líneas 30 y 31 ): Donde dice «entre la primera y la segunda mitad del siglo xvirI», debe decir «en el siglo xvir y en la primera mitad del siglo xviri».

Pág. 168

(Nota 1 del cuadro 18): Donde dice «Edad mínima de los reclutas de 23 años», debe decir «Edad mínima de los reclutas: 23 años».

Pág. 169

(Línea 5): Donde dice «estacionalidad», debe decir *estancamientow.

Pág. 175

(Línea 15): Donde dice «Patterns of Children's Growth», debe decir «Stature and Nutrition».

Pág. 179

(Línea 4): Donde dice «en plazo medio», debe decir «a medio plazo».

Pág. 181

(Línea 6): Donde dice «de nutrición y también», debe decir «de nutrición, es también».

Pág. 184

(Líneas 19 a 21): Donde dice «parecen por el contrario expectativas relacionadas positivamente con las vicisitudes de la mortalidad», debe decir «parece, por el contrario, relacionado positivamente, en contra de lo esperado, con las tendencias de la mortalidad .

(Líneas 38 y 39): Donde dice "y si bien podemos precisarlo», debe decir *y si bien no podemos precisarlo». 
Pág. 185

(Cuadro 19, línea 3): Donde dice «Alimentación animales», debe decir «Alimentación cárnica».

(Cuadro 19, líneas 9 y 10): Donde dice aestacionarion, debe decir «estancamiento».

Pág. 187

(Línea 36): Donde dice «no estuvieron separadas de ellas», debe decir «no estuvieron vinculadas a dicho factor».

Pág. 189

(Línea 20): Donde dice «al menos», debe decir «aunque».

(Línea 23): Donde dice «y de formación», debe decir "y a la formación».

\section{REFERENCIAS BIBLIOGRAFICAS}

ApPleby, Andrew B (1973): «Disease or Famine? Mortality in Cumberland and Westmorland, 1580-1640», The Economic History Review, XXVI, 3, pp. 403-432.

- (1977): «Famine, Mortality and Epidemic Disease: a comment», The Economic History Review, XXX, 3, pp. 508-512.

Barbosa, A., y López Arjona, B. (1935): El paludismo en el primer año de vida, Madrid.

Bellagio Conference (1982): «The Relationship of Nutrition, Disease and Social Conditions: A Graphical Presentation», en Rotberg y Rabb (eds.) (1985). (Las comunicaciones presentadas a la reunión de Bellagio en 1982 fueron publicadas, primero, en The Journal of Interdisciplinary History, XIV, 1983, 2.)

Biraben, Jean-Nöel (1975-76): Les bommes et la peste en France et dans les pays européens et méditerranéens, Paris.

Carmichael, Ann G. (1985): «Infection, Hidden Hunger, and History», en Rotberg y Rabb (eds.) (1985).

DupRQuIER, Jacques (1979): La population française aux XVII et XVIII siècles, París.

Fogel, Robert W., y Engerman, Stanley L. (1981): Tiempo en la cruz. La economía esclavista en los Estados Unidos, Madrid.

Galloway, Patrick R. (1985): "Annual variations in deaths by age, deaths by cause, prices, and weather in London, 1670 to 1830 », Population Studies, 39, 3, pp. 487-505.

- (1988): «Basic patterns in annual variations in fertility, nuptiality, mortality, and prices in pre-industrial Europe», Population Studies, 42, 2, pp. 275-302.

Goubert, Pierre (1952): «En Beauvaisis: problèmes démographiques du $17^{\mathrm{T}}$ siècle», $A n$ nales, ESC, 7, 4, pp. 453-468.

- (1976): Clio parmi les hommes, La Haya-París.

Laslett, Peter (1983): The World We Have Lost -further explored, Cambridge. (Esta es la edición que ha sido traducida al castellano recientemente: El mundo que bemos perdido, explorado de nuevo, Alianza Ed., Madrid, 1987.)

LEE, R. (1981): «Short-term variation: vital rates, prices, and weather», en E. A. Wrigley y R. S. Schofield (1981), cap. 9.

Livi Bacci, M. (1988): Pablación y Alimentación en Europa, Ariel, Barcelona.

McKeown, Thomas (1976): The Modern Rise of Population, Londres (hay versión española: El crecimiento moderno de la población, Antoni Bosch, Barcelona, 1978).

- (1985): «Food, Infection, and Population», en R. I. Rotberg y Th. K. Rabb (eds.) (1985), pp. 29-49.

Meegnan, S. A. (1967): «Malaria Eradication and its Effect on Mortality Levels», Population Studies, XXI, 3, pp. 217-230. 
MerCER, A. J. (1985): «Smallpox and Epidemiological-Demographic Change in Europe: The Role of Vaccination», Population Studies, 39, 2, pp. 287-307.

Meuvret, Jean (1946): «Les crises de subsistances et la démographie de la France d'Ancien Régime», Population, I, 4, pp. 643-650.

Pérez MoReda, Vicente (1980): Las crisis de mortalidad en la España interior (siglos XVI-XIX), Madrid.

- (1984): «Crisis demográficas y crisis agrarias: paludismo y agricultura en España a fines del siglo Xvilı, en Congreso de Historia Rural. Siglos XV al XIX, Madrid, pp. 333.354.

Post, John D. (1976): «Famine, Mortality and Epidemic Disease in the Process of Modernization», The Economic History Review, XXIX, 1, pp. 14-37.

Quinault, R. E. (1976): «Reseña» en The Economic History Review, XXIX, 1, pp. 171 172.

Razzell, P. (1974): «An Interpretation of the Modern Rise of Population in Europe. A Critiques, Population Studies, XXVIII, I.

- (1976): Edward Jenner's Cowpox Vaccine: The History of a Medical Myth, Firle.

- (1977): The Conquest of Smallpox: The Impact of Inoculation on Smallpox Moriality in Eighteenth Century Britain, Firle.

RICHARDS, Toni (1983): "Weather, nutrition and the economy: the analysis of short-run fluctuations in births, deaths and marriages, France 1740-1909», Demography, 20, 2, pp. 197-212.

RotBerg, R. I., y RABB, Th. K. (eds.) (1985): Hunger and History. The Impact of Changing Food Production and Consumption Patterns on Society, Cambridge.

Schofield, R. S. (1985): «The Impact of Scarcity and Plenty on Population Change in England, 1541-1871", en Rotberg y Rabb (eds.) (1985).

SOGNER, Sölvi (1979): «Nature and Dynamics of Crises (including recent crises in developing countries)m, en H. CHARBONNEAU y A. LAROSE (eds.), Les grandes mortalités: étude métbodologique des crises démographiques du passé, Lieja, pp. 311.331.

TAYLOR, Carl E. (1985): «Sinergy among Mass Infections, Famines, and Poverty», en Rotberg y Rabb (eds.) (1985).

WEIR, David R. (1984): «Life under pressure: France and England, 1670-1870», The Journal of Economic History, 44, 1, pp. 27-47.

WRIGLey, E. A., y SCHOFIELD, R. S. (1981): The Population History of England, 15411871. A Reconstruction, Londres.

ZanetrI, Dante E. (1972): La demografia del patriziato milanese nei secoli XVII, XVIII, $X I X$, Pavía. 